Berna Seker Yilmaz ${ }^{\mathrm{a}, \star}$, Neslihan Onenli Mungan, Deniz Kor ${ }^{\mathrm{b}}$, Derya Bulut, Gülşah Seydaoglu, Murat Öktem and Serdar Ceylaner

\title{
Twenty-seven mutations with three novel pathologenic variants causing biotinidase deficiency: a report of 203 patients from the southeastern part of Turkey
}

https://doi.org/10.1515/jpem-2017-0406

Received October 5, 2017; accepted December 7, 2017; previously published online January 20, 2018

\begin{abstract}
Background: Biotinidase deficiency (BD) is an autosomal recessive inborn error of metabolism characterized by neurologic and cutaneous symptoms and can be detected by newborn screening. Newborn screening for BD was implemented in Turkey at the end of 2008.

Methods: In total, 203 patients who were identified among the infants detected by the newborn screening were later confirmed to have BD through measurement of serum biotinidase activity. We also performed BTD mutation analysis to characterize the genetic profile.
\end{abstract}

Results: Twenty-seven mutations were identified. The most commonly found variants were c.1330G $>C$ (p.D444H), c.1595C >T (p.T532M), c.470G >A (p.R157H), and c.198_104delGCGGCTGinsTCC (p.C33Ffs ) with allele frequencies of $0.387,0.175,0.165$ and 0.049 , respectively. Three novel pathogenic and likely pathogenic variants were identified: p.W140* (c.419G>A), p.S319F (c.956C >T) and p.L69Hfs ${ }^{\star} 4$ (c.192_193insCATC). We also identified three mutations reported in just one patient in the past (p.V442Sfs ${ }^{\star} 59$ [c.1324delG], p.H447R [c.1340A >G] and p.198delV [c.592_594delGTC]). Although all of the patients were asymptomatic under the treatment of biotin, only one patient, who had the novel c.419G $>\mathrm{A}$ homozygous mutation became symptomatic during an episode of acute gastroenteritis with a presentation of ketosis and

aPresent address: Department of Pediatric Metabolism, Mersin City Hospital, Mersin, Turkey

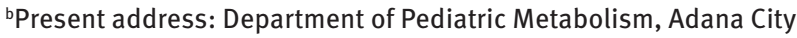
Hospital, Adana, Turkey

*Corresponding author: Berna Seker Yilmaz, Department of Pediatric Metabolism, Mersin City Hospital, Mersin, Turkey,

Phone: +905439699013, Fax: +903223386931,

E-mail: berna_seker@yahoo.co.uk; and Department of Pediatric Metabolism, University Hospital Cukurova, Adana, Turkey

Neslihan Onenli Mungan, Deniz Kor and Derya Bulut: Department of Pediatric Metabolism, University Hospital Cukurova, Adana, Turkey Gülşah Seydaoglu: Department of Biostatistics, University Hospital Cukurova, Adana, Turkey

Murat Öktem: Duzen Biochemistry Laboratories, Ankara, Turkey

Serdar Ceylaner: Intergen Genetics Laboratories, Ankara, Turkey metabolic acidosis. Among the screened patients, 156 had partial and 47 had profound BD.

Conclusions: We determined the mutation spectra of $\mathrm{BD}$ from the southeastern part of Turkey. The results of this study add three more mutations to the total number of mutations described as causing BD.

Keywords: biotinidase; novel mutation; partial; profound.

\section{Introduction}

Biotinidase deficiency (BD) (MIM \#253260) is an autosomal recessive disease in which the cleavage and recycling of biotin from biocytin or biotinylated peptides are impaired [1]. This leads to a deficiency in biotin-dependent enzymes, such as propionyl-CoA carboxylase, betamethyl-crotonyl-CoA carboxylase, pyruvate carboxylase, and acetyl-CoA carboxylase. It also plays an important role in the biosynthesis of fatty acids, gluconeogenesis, the tricarboxylic acid cycle, anaplerosis and pleiotropic gene regulation, and carbohydrate metabolism [2]. Untreated individuals will likely develop neurological symptoms, such as hypotonia, seizures, ataxia, developmental delay, sensorineural hearing loss and optic atrophy, immune deficiency, and cutaneous symptoms, such as skin rash, conjunctivitis, alopecia with accompanying ketolactic acidosis and organic aciduria [3, 4]. BD is classified as either profound or partial based on the serum biotinyl-hydrolase enzyme activity, $<10 \%$ and $10-30 \%$ of mean normal serum activity, respectively [5]. Long-term follow-up studies indicate that the majority of patients with partial BD do not exhibit symptoms unless stressed by infections, prolonged fasting, etc. [6, 7]. Symptoms can be improved and the progression halted with pharmacological doses of oral biotin. Early treatment with pharmacological doses of oral biotin completely prevents clinical disease and symptoms can be improved; however, some symptoms such as vision and hearing problems and developmental delay may be irreversible [8]. There is still no consensus on the need for the treatment of partial BD [9].

Newborn screening for BD was implemented in the last months of 2008 in Turkey. Our clinic is the main metabolic center in the southeastern part of Turkey. Because 
of the complex ethnic structure of the population in the southeastern part of Turkey, we sought to determine the overall incidence of BD. We also performed BD mutation analysis in search of a possible common genotype. It has also been reported that, in some cases, enzymatic activity does not adequately differentiate partial deficiency from heterozygosity for profound deficiency; therefore, mutation analysis is necessary to confirm the diagnosis [9].

The human biotinidase gene is located on chromosome 3p25 and consists of four exons with a total length of $1629 \mathrm{bp}$ and encodes a mature protein of 543 amino acids [10]. To date, more than 140 mutations causing profound BD have been described [11]. Genotype-phenotype correlation is still not well established. This report summarizes the results of newborn biotinidase screening from April 2013 through April 2016, and BD mutation analysis results for 203 Turkish cases.

\section{Subjects and methods}

\section{Subjects}

In total, 203 newborns from the southeastern part of Turkey were diagnosed as BD according to the newborn screening conducted between April 2013 and April 2016.

\section{Biotinidase activity measurement}

Biotinidase activity was initially measured in dried blood spots (DBS) via colorimetric enzyme assay on Guthrie cards. A serum sample was requested if the initial sample showed a positive result, which means that biotinidase activity was under $30 \%$ of the mean of normal controls. Quantitative biotinidase activity measurement and molecular analysis of the BTD gene were performed on these subjects.

Biotinidase activity was measured in DBS (initial sample) on Guthrie cards as reported previously [12]. If the initial sample showed a positive result, the enzyme activity was measured in the serum of positive cases using a colorimetric enzyme activity method with biotin 4-aminobenzoic acid as the substrate. A Biotek Synergy H1 spectrophotometer (BioTek Instruments, Winooski, VT, USA) was used at $546 \mathrm{~nm}$ for the measurement of biotinidase activity. The cut-off level for biotinidase enzyme activity is $3.5-13.8 \mathrm{U} / \mathrm{L}$ in our laboratory. $\mathrm{BD}$ is classified as profound and partial deficiency based on the serum biotinidase enzyme activity, $<10 \%$ and $10-30 \%$ of mean normal serum activity, respectively.

After obtaining an informed consent from both parents, blood samples were collected from BD patients.

\section{Molecular analysis}

Molecular analysis of the BTD gene was done at Intergen Genetics Laboratory, Ankara by the next-generation sequencing (NGS) method using an MiSeq instrument (Illumina Inc., San Diego, CA, USA) and in-house designed primers. After polymerase chain reaction (PCR) amplification, the libraries were prepared with the NexteraXT kit (Illumina Inc., San Diego, CA, USA). Sequences were aligned to the hy19 genome within MiSeq Reporter software (Illumina Inc., San Diego, CA, USA). Visualization of the data was performed with IGV 2.3 (Broad Institute, CA, USA) software. NM_001281724.2 was used for naming of the mutations. HGMD, ClinVar, LOVD mutation databases and a PubMed search was done for all mutations and a mutation that was not present in those databases was classified as "novel". In silico analysis was done using Mutation Taster, Mutation Assessor, FATHMM, FATHMM-MKL, MetaSVM, MetalR, Polyphen2, Provean, LRT, GM12878, SIFT, phyloP20way, phyloP100way, phastCons20way, phastCons100way, SiPhy29way online tools.

\section{Results}

Neonates froom the southeastern part of Turkey were screened for $\mathrm{BD}$ in the pediatric metabolism and nutrition clinic of the Cukurova University between April 2013 and April 2016. Two hundred and three patients with BD were included in the study. Although 125 patients (61.5\%) had consanguineous parents, only 114 families have homozygote mutations. In nine consanguineous families, we detected compound heterozygote mutations. In total, we detected compound heterozygote mutations in 89 of 203 patients. This is one of the most important presentations of a very high carrier frequency rate in the population. We have identified four patients with three novel mutations in the BTD gene (p.W140* [c.419G $>$ A], p.S319F [c.956C >T], p.L69Hfs ${ }^{\star} 24$ [c.192_193insCATC]). We also identified three mutations reported in just one patient in the past (p.V442Sfs`59 [c.1324delG]/p.H447R [c.1340A>G]/ p.198delV [c.592_594delGTC]). p.V442Sfs`59 (c.1324delG) mutation was reported in another Turkish patient [13].

While 47 patients have a profound deficiency, 156 of them have partial enzyme deficiency. The mutational screening revealed 27 different mutations, 24 of them were reported in the previous studies, three were novel (Table 1 ). Demographic and enzymatic features of the patients with novel and rarely reported mutations are shown in Table 2. Their references and definitions in the American College of Medical Genetics and Genomics (ACMG) standards and guidelines are given in Table 3.

According to the distribution of mutations, 19 were missense, two were frameshift insertions, one was a frameshift deletion, one was a in-frame deletion, two were nonsense mutations and one was a gross deletion. The details are given in Table 1.

All patients except one, identified as having BD by newborn screening and who were immediately started 
Table 1: Mutations causing biotinidase deficiency (NM_001281724.2).

\begin{tabular}{|c|c|c|c|c|c|c|c|c|}
\hline $\begin{array}{l}\text { Protein } \\
\text { change }\end{array}$ & Nucleotide change & Note & Exon & $\begin{array}{r}\text { Allele } \\
\text { no }\end{array}$ & Type of mutation & $\begin{array}{r}\text { Allele } \\
\text { frequency }\end{array}$ & $\%$ & $\begin{array}{l}\text { Number of } \\
\text { newborns }\end{array}$ \\
\hline p.D444H & c. $1330 \mathrm{G}>\mathrm{C}$ & & 4 & 157 & Missense & 0.387 & 38.7 & 115 \\
\hline p.T532M & c. $1595 C>T$ & & 4 & 71 & Missense & 0.175 & 17.5 & 71 \\
\hline p.R157H & c. $470 \mathrm{G}>\mathrm{A}$ & & 4 & 67 & Missense & 0.165 & 16.5 & 47 \\
\hline p.C33Ffs $* 36$ & c.98-104delGCGGCTGinsTCC & & 2 & 20 & Frame-shift insertion & 0.049 & 4.9 & 16 \\
\hline p.R79C & c. $235 \mathrm{C}>\mathrm{T}$ & & 2 & 14 & Missense & 0.034 & 3.4 & 10 \\
\hline p.C418S & c. $1253 \mathrm{G}>\mathrm{C}$ & & 4 & 12 & Missense & 0.030 & 3.0 & 8 \\
\hline p.Q456H & C. $1368 \mathrm{~A}>\mathrm{C}$ & & 4 & 11 & Missense & 0.027 & 2.7 & 6 \\
\hline p.C186Y & c. $557 \mathrm{G}>\mathrm{A}$ & & 4 & 10 & Missense & 0.025 & 2.5 & 7 \\
\hline \multirow[t]{2}{*}{ p.V442Sfs*59 } & c.1324delG & Rarely reported & 4 & 6 & Frame-shift deletion & 0.015 & 1.5 & 6 \\
\hline & Exon1 deletion & & 1 & 6 & Gross deletion & 0.015 & 1.5 & 3 \\
\hline p.Y454C & c. $1361 \mathrm{~A}>\mathrm{G}$ & & 4 & 5 & Missense & 0.012 & 1.2 & 5 \\
\hline p.Y57* & c. $171 \mathrm{~T}>\mathrm{G}$ & & 4 & 4 & Nonsense & 0.010 & 1.0 & 3 \\
\hline p.W140* & c. $419 \mathrm{G}>\mathrm{A}$ & Novel & 3 & 3 & Nonsense & 0.007 & 0.7 & 2 \\
\hline p.R209C & c. $625 \mathrm{C}>\mathrm{T}$ & & 4 & 3 & Missense & 0.007 & 0.7 & 3 \\
\hline p.L69Hfs $* 24$ & c.192_193insCATC & Novel & 4 & 3 & Frame-shift insertion & 0.007 & 0.7 & 3 \\
\hline p.S319F & c. $956 C>T$ & Novel & 4 & 2 & Missense & 0.005 & 0.5 & 1 \\
\hline p.G445R & c. $133 \mathrm{G}>\mathrm{A}$ & & 2 & 2 & Missense & 0.005 & 0.5 & 1 \\
\hline p.G114V & c. $341 \mathrm{C}>\mathrm{T}$ & & 3 & 2 & Missense & 0.005 & 0.5 & 2 \\
\hline p.Y93C & c. $278 \mathrm{~A}>\mathrm{G}$ & & 2 & 1 & Missense & 0.002 & 0.2 & 1 \\
\hline p.R211C & $\mathrm{c} .631 \mathrm{C}>\mathrm{T}$ & & 4 & 1 & Missense & 0.002 & 0.2 & 1 \\
\hline p.Q511E & c. $1531 C>G$ & & 6 & 1 & Missense & 0.002 & 0.2 & 1 \\
\hline p.P497S & c. $1489 C>T$ & & 4 & 1 & Missense & 0.002 & 0.2 & 1 \\
\hline p.P369L & c. $1106 C>T$ & & 4 & 1 & Missense & 0.002 & 0.2 & 1 \\
\hline p.H447R & c. $1340 A>G$ & Rarely reported & 4 & 1 & Missense & 0.002 & 0.2 & 1 \\
\hline p.A171T & c. $511 \mathrm{G}>\mathrm{A}$ & & 4 & 1 & Missense & 0.002 & 0.2 & 1 \\
\hline p.198delV & c.592_594delGTC & Rarely reported & 4 & 1 & In-frame deletion & 0.002 & 0.2 & 1 \\
\hline
\end{tabular}

Table 2: Demographic features and enzyme levels of the patients with novel and rarely reported mutations.

\begin{tabular}{|c|c|c|c|}
\hline Patient no & Enzyme activity & Allele 1 & Allele 2 \\
\hline 1 & $<0.5$ & p.S319F (c.956C>T) & p.S319F (c.956C>T) \\
\hline 2 & 1.92 & p.198delV (c.592_594delGTC) & p.D444H (c.1330G >C) \\
\hline 3 & 0.5 & p.V442Sfs*59 (c.1324delG) & p.D444H (c.1330G>C) \\
\hline 4 & 1.9 & p.V442Sfs*59 (c.1324delG) & p.D444H (c.1330G>C) \\
\hline 5 & 2.01 & p.V442Sfs*59 (c.1324delG) & p.D444H (c.1330G>C) \\
\hline 6 & 1.3 & p.V442Sfs*59 (c.1324delG) & p.D444H (c.1330G>C) \\
\hline 7 & 2.1 & p.V442Sfs*59 (c.1324delG) & p.D444H (c.1330G>C) \\
\hline 8 & 1.4 & p.V442Sfs*59 (c.1324delG) & p.D444H (c.1330G>C) \\
\hline 9 & 0.9 & p.W140* (c.419G $>A)$ & p.W140* (c.419G>A) \\
\hline 10 & 1.75 & p.W140* (c.419G >A) & p.R157H (c.470G>A) \\
\hline 11 & 2.2 & p.L69Hfs*24 (c.192_193insCATC) & p.D444H (c.1330G>C) \\
\hline 12 & 2.3 & p.L69Hfs*24 (c.192_193insCATC) & p.D444H (c.1330G>C) \\
\hline 13 & 2.1 & p.L69Hfs*24 (c.192_193insCATC) & p.D444H (c.1330G>C) \\
\hline 14 & 2.3 & p.H447R (c.1340A>G) & p.D444H (c.1330G>C) \\
\hline
\end{tabular}

on biotin supplementation have been asymptomatic on biotin therapy. One patient who was homozygous for the novel p.W140* mutation presented with metabolic acidosis and ketosis during an episode of acute gastroenteritis under biotin treatment. With biotin dose increment, the symptoms disappeared and an asymptomatic course of treatment was provided.

\section{Discussion}

$\mathrm{BD}$ is a rare recessive inherited metabolic disease characterized by phenotypic and genotypic heterogeneity. The incidence of profound BD patients is 1:112,271 births and the incidence of partial BD cases is 1:129,281 births [14]. The estimated combined incidence of profound and 
Table 3: Novel and rarely reported mutations in our study.

\begin{tabular}{|c|c|c|c|c|}
\hline & & & Reference & $\begin{array}{l}\text { ACMG standards } \\
\text { and guidelines } \\
\text { (http://www.nature. } \\
\text { com/gim/journal/ } \\
\text { v17/n5/full/ } \\
\text { gim201530a.html) }\end{array}$ \\
\hline p.W140* & c. $419 \mathrm{G}>\mathrm{A}$ & Novel & & Very strong: PVS1 \\
\hline p.L69Hfs*24 & c.192_193insCATC & Novel & & Very strong: PVS1 \\
\hline p.S319F & c.956C $>\mathrm{T}$ & Novel & & $\begin{array}{l}\text { In silico analysis } \\
\text { data were given } \\
\text { below }\end{array}$ \\
\hline p.V442Sfs*59 & c.1324delG & Rarely reported & $\begin{array}{l}\text { http://www.turkiyeklinikleri.com/article/tr-yenidogan- } \\
\text { tarama-testinde-biotinidaz-eksikligi-saptanan-bir- } \\
\text { olgu-68569.html }\end{array}$ & Very strong: PVS1 \\
\hline p.H447R & c. $1340 A>G$ & Rarely reported & $\begin{array}{l}\text { http://download.springer.com/static/pdf/806/art\%2 } \\
\text { 53A10.1186\%252Fs12859-016-1286-0.pdf?originUrl } \\
\text { =http\%3A\%2F\%2Fbmcbioinformatics.biomedcentral. } \\
\text { com\%2Farticle\%2F10.1186\%2Fs12859-016-1286-0\&token } \\
\text { 2=exp=1485932350 acl=\%2Fstatic\%2Fpdf\%2F806\%2Fart } \\
\% 25253 \mathrm{~A} 10.1186 \% 25252 \mathrm{Fs} 12859-016-1286-0 . p d f * \sim \mathrm{hmac} \\
=4 \mathrm{f3} 264 \mathrm{ecd} 7370018 \mathrm{e} 1 \mathrm{e} 0 \mathrm{e} 59 \mathrm{~d} 557 \mathrm{fcf} 1 \text { b0663a0d1ddd1395 } \\
\text { 4327e89832b960dce }\end{array}$ & \\
\hline p.198delV & c.592_594delGTC & Rarely reported & $\begin{array}{l}\text { rs } 397514373 \\
\text { ClinVar RCV000021945 }\end{array}$ & \\
\hline
\end{tabular}

partial BD is 1:60,089 births [15]. The incidence of BD in Turkey is 1:7116 [16]. It is expected that the incidence will be even higher in our clinic in the southeastern part of Turkey due to the relatively higher frequency of consanguineous marriages.

Currently, 165 mutations of the BTD gene have been reported [17]. As was recently reported, the association between the BTD genotype and biotinidase activity is not certain $[2,18]$. As established, p.D444H is the most common mutation in our patients and as reported in the previous studies [19], the majority of children with partial deficiency had at least one allele with the p.D444H mutation [20]. In the present study, there is a contradiction between the observed and expected biochemical phenotype in the same cases; in this context we would like to highlight patients who were compound heterozygous or homozygous for the $\mathrm{D} 444 \mathrm{H}$ mutation with lower residual biotinidase activity levels. This controversy suggests that there are some other epigenetic and biochemical factors involved. It should be considered that the biotinidase activity expressed in vitro might be different from that in vivo, so it is better to make an estimation by the study of protein expression.

The measurement of enzyme activity is important in the diagnosis of BD. However, mutation screening may confirm the diagnosis, may prevent the misdiagnosis due to biochemical and analytical methods and may explain the possible effects of homozygous mutations on enzyme activity especially in the populations with higher consanguinity rates, which could enhance the understanding of genotype-phenotype correlation.

As newborn screening for $\mathrm{BD}$ is incorporated into the newborn screening program in Turkey, very rare symptomatic children with the disorder have been identified. It has been proposed that children with some residual enzyme activity may have a milder clinical course than those with no measurable biotinidase activity and may have a lower requirement for biotin supplementation for treatment [7]. In this report, only one patient was symptomatic under biotin treatment during an acute gastroenteritis episode. Clinical symptoms disappeared with an increase in biotin dosage. It is important that all children, even with profound BD, should be treated with adequate biotin therapy [21].

In conclusion, we determined the mutation spectrum causing BD combined with biochemical analysis in patients from the southeastern part of Turkey. The results of this work add three novel mutations (c.956C > T [p.S319F], c.192_193insCATC [p.L69Hfs $\left.{ }^{\star} 24\right]$, c.419G $>$ A [p.W140^]) to the number of mutations described previously. We also detected three mutations reported in just one case previously (p.V442Sfs`59 [c.1324delG]/p.H447R [c.1340A>G]/ p.198delV [c.592_594delGTC]). p.V442Sfs`59 (c.1324delG) mutation was reported in another Turkish patient (Table 3). 
This provides a clinical support for those variations for a description as disease-causing variants. The results of this study strongly support the existing information on DNA variation among populations and ethnic groups.

Author contributions: All the authors have accepted responsibility for the entire content of this submitted manuscript and approved submission.

Research funding: None declared.

Employment or leadership: None declared.

Honorarium: None declared.

Competing interests: The funding organization(s) played no role in the study design; in the collection, analysis, and interpretation of data; in the writing of the report; or in the decision to submit the report for publication.

\section{References}

1. Wolf B. Biotinidase: its role in biotinidase deficiency and biotin metabolism. J Nutr Biochem 2005;16:441-5.

2. Thodi G, Schulpis KH, Molou E, Georgiou V, Loukas LK, et al. High incidence of partial biotinidase deficiency cases in newborns of Greek origin. Gene 2013;524:361-2.

3. Salbert BA, Pellock JM, Wolf B. Characterization of seizures associated with biotinidase deficiency. Neurology 1993;43:1351-4.

4. Wolf B, Heard GS, Weissbecker KA, McVoy JR, Grier RE, et al. Biotinidase deficiency: Initial clinical features and rapid diagnosis. Ann Neurol 1985;18:614-7.

5. Ohlsson A, Guthenberg C, Holme E, von Döbeln U. Profound biotinidase deficiency: a rare disease among native Swedes. J Inherit Metab Dis 2010;33:175-80.

6. McVoy JR, Levy HL, Lawler M. Partial biotinidase deficiency: clinical and biochemical features. J Pediatr 1990;116:78-83.

7. Möslinger D, Stockler-Ipsiroglu S, Scheibenreiter S. Clinical and neuropsychological outcome in 33 patients with biotinidase deficiency ascertained by nationwide newborn screening and family studies in Austria. Eur J Pediatr 2001;160:277-82.

8. Heard GS, Wolf B, JeVerson RG, Weissbecker KA, Nance VE, et al. Screening for biotinidase deficiency: results of a 1-year pilot study. J Pediatr 1986;108:40-6.

9. Wolf $B$. Clinical issues and frequent questions about biotinidase deficiency. Mol Genet Metab 2010;100:6-13.

10. Knight HC, Reynolds TR, Meyers GA, Pomponio RJ, Buck GA, et al. Structure of the human biotinidase gene. Mamm Genome 1998;9:327-30.

11. Pindolia K, Jordan M, Wolf B. Analysis of mutations causing biotinidase deficiency. Hum Mutat 2010;31:983-91.

12. Heard GS, SecorMcVoy JR, Wolf BA. A screening method for biotinidase deficiency in newborns. Clin Chem 1984;30:125-7.

13. Kavasoglu AN, Onay H, Kose M, Durmaz A, Kalkan S, et al. A case diagnosed with biotinidase deficiency in newborn screening test. Turkiye Klinikleri J Pediatr 2014;23:49-52.

14. Zempleni J, Hassan YI, Wijeratne SS. Biotin and biotinidase deficiency. Exp Rev Endocrinol Metab 2008;3:715-24.

15. Wolf B. Disorders of biotin metabolism. In: Scriver CR, Baudet AL, Sly WS, Valle D, editors. The metabolic and molecular bases of inherited disease. New York: McGraw-Hill, 2001:3935-62.

16. Karaca M, Ozgül RK, Unal O, Yucel-Yilmaz D, Kılıc M, et al. Detection of biotinidase gene mutations in Turkish patients ascertained by newborn and family screening. Eur J Pediatr 2015;174:1077-84.

17. Procter M, Wolf $B$, Mao R. Forty-eight novel mutations causing biotinidase deficiency. Mol Genet Metab 2016;117:369-72.

18. Neto EC, Schulte J, Rubim R, Lewis E, DeMari J, et al. Newborn screening for biotinidase deficiency in Brazil: biochemical and molecular characterizations. Braz J Med Biol Res 2004;37:295-9.

19. Pomponio RJ, Coskun T, Demirkol M, Tokatli A, Ozalp I, et al. Novel mutations cause biotinidase deficiency in Turkish children. J Inherit Metab Dis 2000;23:120-8.

20. Katie L, Swango MD, Huner G, Pronicka E, Sykut-Cegielska JJ, et al. Partial biotinidase deficiency is usually due to the $\mathrm{D} 444 \mathrm{H}$ mutation in the biotinidase gene. Hum Genet 1998;102:571-5.

21. Wolf $B$. Children with profound biotinidase deficiency should be treated with biotin regardless of their residual enzyme activity. Eur J Pediatr 2002;161:167-8. 